\title{
A Multi-Vehicle Testbed for Underwater Motion Coordination
}

\author{
Nitin Sydney \\ Department of Aerospace \\ Engineering \\ University of Maryland, \\ College Park, MD 20742 \\ nsydney@umd.edu
}

\author{
Seth Napora \\ Department of Aerospace \\ Engineering \\ University of Maryland, \\ College Park, MD 20742 \\ snapora@umd.edu
}

\author{
Derek A. Paley \\ Department of Aerospace \\ Engineering \\ University of Maryland, \\ College Park, MD 20742 \\ dpaley@umd.edu
}

\begin{abstract}
The Synthetic Collective Unmanned Underwater Laboratory (SCUUL) testbed is a multi-vehicle testbed that is used to evaluate the performance of underwater motion coordination algorithms in a dynamic environment. The SCUUL testbed consists of six propellor-driven vehicles, a 367,000 gallon tank, and an underwater motion capture system. The tank is the Neutral Buoyancy Research Facility (NBRF) located at the University of Maryland and operated by the Collective Dynamics and Control Laboratory (CDCL) and the Space Systems Laboratory. The motion capture is a state-of-the-art system developed by Qualisys in Gothenburg, Sweden. Initial results have shown the capabilities of the separate components of SCUUL and its ability to test and evaluate a multitude of motion coordination algorithms in a laboratory environment.
\end{abstract}

\section{Keywords}

Underwater vehicles, cooperative control, motion coordination

\section{INTRODUCTION}

Testing of cooperative motion algorithms can be difficult in a real world underwater environment. Issues such as limited communication, environmental setbacks, deployment costs and many other problems can be detrimental to vehicle testing and the implementation of algorithms. Verifying the performance of the algorithms can be challenging, if not impossible, in such an environment. There is a need for a testbed in which motion coordination algorithms for underwater vehicles can be tested without the challenges listed above.

The Synthetic Collective Unmanned Underwater Laboratory at the University of Maryland provides a controlled environment where algorithms for underwater motion coordination can be tested and verified while avoiding many of the

Permission to make digital or hard copies of all or part of this work for personal or classroom use is granted without fee provided that copies are not made or distributed for profit or commercial advantage, and that copies bear this notice and the full citation on the first page. To copy otherwise, to republish, to post on servers or to redistribute to lists, requires prior specific permission and/or a fee.

PerMIS '10 September 28-30, 2010, Baltimore, MD, USA

Copyright 2010 ACM 978-1-4503-0290-6-9/28/10 ...\$10.00. problems encountered in real world environments. SCUUL is a multi-vehicle testbed consisting of six propellor-driven unmanned underwater vehicles (UUVs) operated at the University of Maryland's Neutral Buoyancy Research Facility (NBRF). The NBRF is a 367,000 gallon tank filled with clear, filtered water that is equipped with a state-of-the-art underwater motion capture system. The motion capture system is used for two purposes: to stream data in real time to the UUVs for feedback control and to verify cooperative motion algorithms.

The objective of SCUUL is to apply dynamical systems theory to implement and verify nonlinear motion coordination algorithms for underwater vehicles. The focus of this paper is to show the utility of the SCUUL testbed for UUV navigation, sampling, performance and control. Examples of such algorithms are shown in [4],[1],[3], and [2].

Section 2 will describe the testbed and it's capabilities in detail. Section 3 will describe the preliminary results and ongoing work of the project.

\section{THE SCUUL TESTBED}

This section describes the SCUUL testbed, which consists of six propellor driven submarines, the NBRF and an underwater motion capture system.

\subsection{The UUVs}

The SCUUL testbed includes six propellor-driven UUVs as shown in Figure 1. The submarines are 1:60 scale models of the USS Albacore. They are RC kits purchased from Mike's Sub Works LLC that can be operated using a standard $\mathrm{RC}$ radio transmitter.

The outer hull consists of a nose cone, tail cone, a mast, and a two-part main section. Attached to the tail cone are four control surfaces: two rudders and two elevators. The interior of the submarines contains two pressure vessels. The first pressure vessel houses the battery which powers the submarine during operation. The second pressure vessel, called the main pressure vessel (MPV), contains all the electronics. This includes two servos, a DC motor, a Viper speed controller, an automatic depth controller, a HITEC Laser4 radio control system, and a voltage regulator to manage power fluctuations. Both the MPV and battery compartments are sealed by two endcaps with O-rings. The interior of the submarines is shown in Figure 2.

The depth of the submarines is regulated by an automatic depth controller, which controls the elevators. The desired depth is adjustable using a potentiometer. The yaw motion 


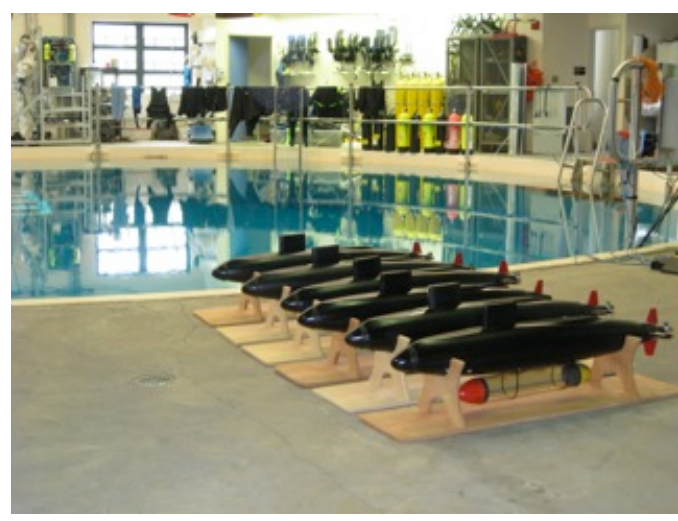

Figure 1: The SCUUL testbed.

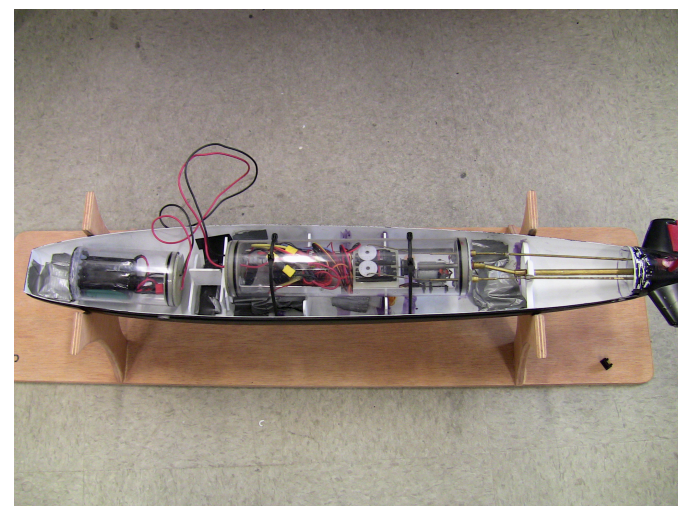

Figure 2: The interior view showing the battery compartment and the main pressure vessel.

of the UUV is controlled either by human in teleoperation using an RC transmitter, or autonomously by an autopilot, which is described below.

Attached to the bottom of each submarine is an autopilot unit $(\mathrm{AU})$ that provides onboard feedback control to the submarines to close the loop on the second order dynamics of the rudder. In the AU there are two main assemblies. The first assembly consists of the components needed for wireless communication. This includes a HITEC Laser4 radio control system to respond to commands from the top side and a modified radio transmitter to communicate from the $\mathrm{AU}$ to the submarines radio receiver. The second assembly consists of a single-axis gyroscope mounted vertically a PIC micro controller. The gyroscope is attached to the PIC using an SPI connection and provides the submarines turning rate up to $\pm 300^{\circ} / \mathrm{sec}$. The PIC relays the turning commands to the submarine by the means of four digital potentiometers, which are meant to take the place of the physical potentiometers in the original transmitter.

Due to a limited amount of space in the AU pressure vessel, some of the components were printed onto a custom circuit board. This reduces the amount of wiring in the AU and hence increases the available space. The components on the circuit board include the PIC microcontroller, the one-axis gyroscope and the four digital potentiometer. The printed circuit board is shown below.

The depth controller enables the SCUUL testbed to test

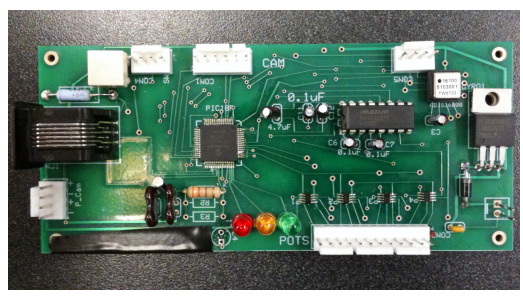

Figure 3: The custom circuit board containing a PIC microcontroller, a single-axis gyroscope, and four digital potentiometers.

planar coordination algorithms. For the algorithms SCUUL is currently testing, the AU needs to know the desired heading rate. The desired heading rate is sent to the AU via the underwater motion capture system (described in Section 2.3). The desired heading rate is calculated using nonlinear control laws developed for a reduced order model [4]. The AU uses the desired heading rate, $\dot{\theta}_{d}$, and the current heading rate, $\dot{\theta}$, given by the gyroscope to close the loop on the rudder dynamics with a proportional gontroller controller, given below

$$
u=-K\left(\dot{\theta}_{d}-\dot{\theta}\right)
$$

where $K>0$ is the proportional gain. Figure 4 shows the AU attached to the bottom of a SCUUL UUV.

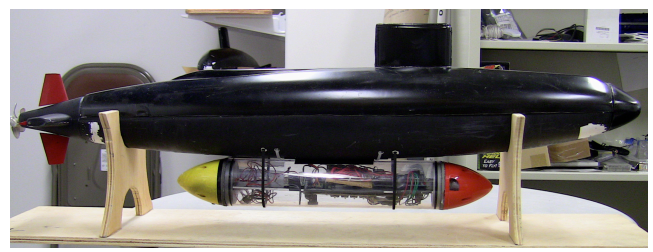

Figure 4: The autopilot unit attached to the bottom of a SCUUL submarine.

\subsection{NBRF}

The NBRF is a 367,000 gallon tank containing clear, filtered water kept at a nominal temperature of $90^{\circ} \mathrm{F}$. The tank is $50 \mathrm{ft}$ across and $25 \mathrm{ft}$ deep making it the largest neutral buoyancy facility at any university worldwide. The CDCL uses NBRF as a dive tank to conduct all tests of the SCUUL UUV fleet. The NBRF is shown in Figure 1.

Research is currently being conducted on methods to generate water currents in the tank. One such example of this is a 3600 gallon-per-hour waterfall pump that can be used in NBRF with the submarines. This pump can generate significant flows that allow for a laboratory-scale test of control algorithms in a dynamic environment. The waterfall pump used by the CDCL is shown in Figure 5.

\subsection{Underwater Motion Capture System}

The NBRF is equipped with a state-of-the-art underwater motion capture system. This system consists of twelve underwater cameras designed and manufactured by Qualysis, based in Gothenburg, Sweden. The camera placements in the tank are shown in Figure 6 below.

There are eight cameras at anupper level and four on a lower level. The cameras are angled to maximize the cov- 


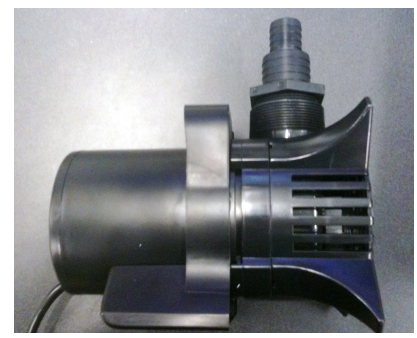

Figure 5: Waterfall pump used to generate underwater currents.

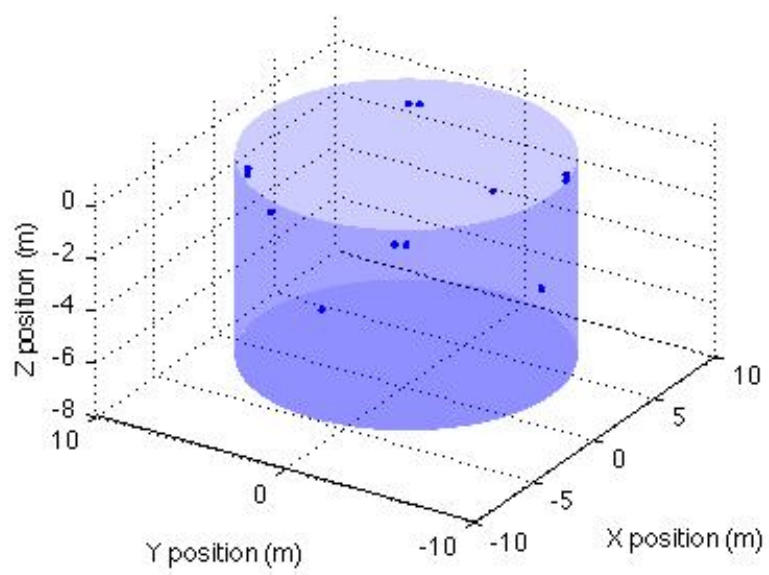

Figure 6: Locations of the motion capture cameras

erage of the system. The upper and lower cameras are two and fifteen feet in depth, respectively, from the surface.

The upper-level cameras are mounted in pairs while the lower cameras are mounted individually as seen in Figure 6. One of the upper level mounts is shown below in Figure 7 . The cameras are all connected to a single operating computer via four ethernet switches. Data streams to the computer from each camera at a nominal rate of $20 \mathrm{~Hz}$, but can be adjusted to capture at up to $100 \mathrm{~Hz}$.

The motion capture cameras have two modes of operation. The first mode is video capture. In this mode the cameras behave like an ordinary video camera, with the footage being stored on the operating computer. The second mode is marker tracking. In this mode, the cameras track reflective markers specially made for the purpose of underwater tracking. The data given is the $3 \mathrm{D}$ position of every marker, as well as a residual for the error in the estimate. Depending on the calibration, this error is usually between $0.5-1.0 \mathrm{~cm}$. When there are three or more markers on a single object, the markers can be formed into a rigid body. The data given for rigid-body tracking is the $3 \mathrm{D}$ position of the center of mass of the object, the associated 3-2-1 Euler angles for the rotation, and the rotation matrix. This mode also has the capability of streaming data in real-time.

Using the motion capture system in real-time mode allows information to be sent to the AU and used by the inner-loop controller to perform closed-loop feedback control for each of the vehicles in the fleet. This architecture can be utilized

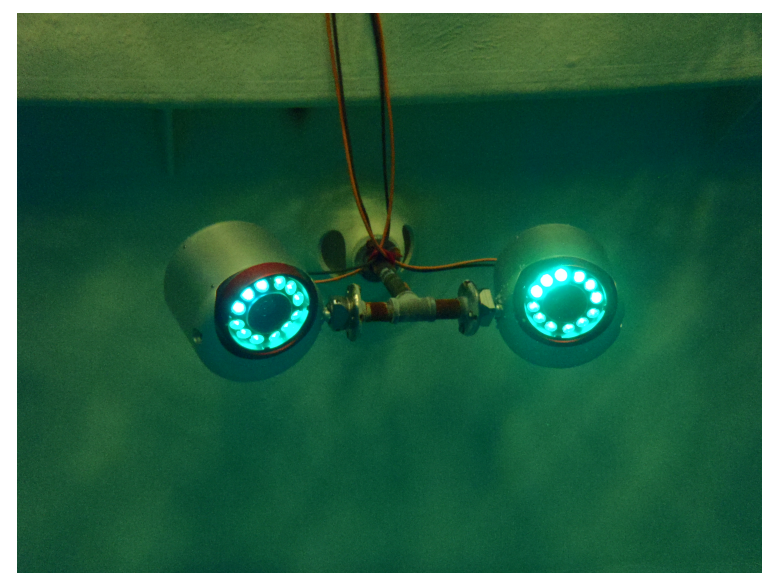

Figure 7: Upper level mount of two Qualisys motion capture cameras.

to test a multitude of coordination algorithms, such as those developed in [1], [3], and a multitude of other algorithms.

The UUVs in the SCUUL fleet each have six markers at varying positions on the outer hull. This ensures that the software will not confuse the submarines and that occluded markers will not have a significant effect on the tracking capabilities of the system. The markers are $30 \mathrm{~mm}$ in diameter so that the Qualisys cameras can see them from across the tank. Figure 8 shows one of the SCUUL UUVs with markers attached to it.

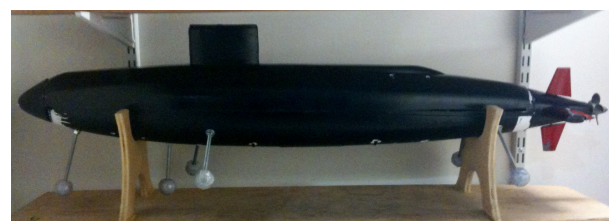

Figure 8: A SCUUL UUV equipped with reflective markers.

The real-time data is sent to the AU using a PCTx interface. The motion capture system streams data to a control computer, which is connected to the PCTx interface. The PCTx interface interprets the data and sends it to a transmitter, which then transfers the data to the AU. Since the transmitter uses standard RF communication there is a limited line of sight through water in which the signal will successfully transmit, however, the conditions at the NBRF allow penetration of the signal to at least the depth of the lower level cameras.

A schematic of the SCUUL architecture is shown in Figure 9. The Qualisys data is sent from the control computer to the PCTx box, which transmits the desired turning rate to the AU of each submarine. The AU calculates the control signal needed to control the loop on the second-order dynamics and transmits it to the submarine it is attached to.

\section{PRELIMINARY RESULTS AND ONGO- ING WORK}

This section describes the progress made in the SCUUL testbed so far and describes our current efforts to improve 


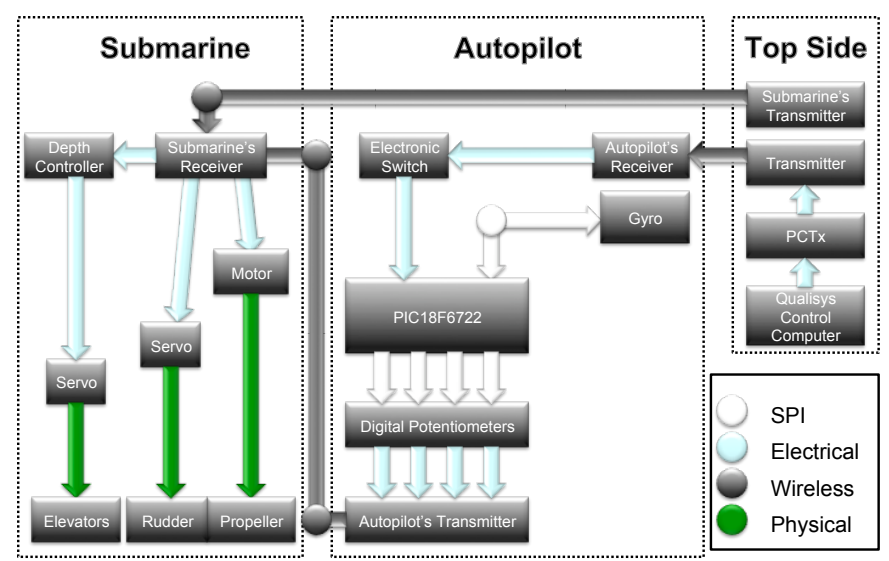

Figure 9: Schematic of topside interface and the submarine/autopilot architecture.

the system.

\subsection{Results}

The performance of the proportional controller for the rudder dynamics has been evaluated. Figure 10 shows the performance of the controller to an impulse starting at approximately 0.25 seconds. The error settles to a nominal value of zero within only one second, which is an acceptable settling time for the purposes of the control laws currently being verified by SCUUL. The settling time, and peak response, can be adjusted according to the need of the algorithm that is under testing.

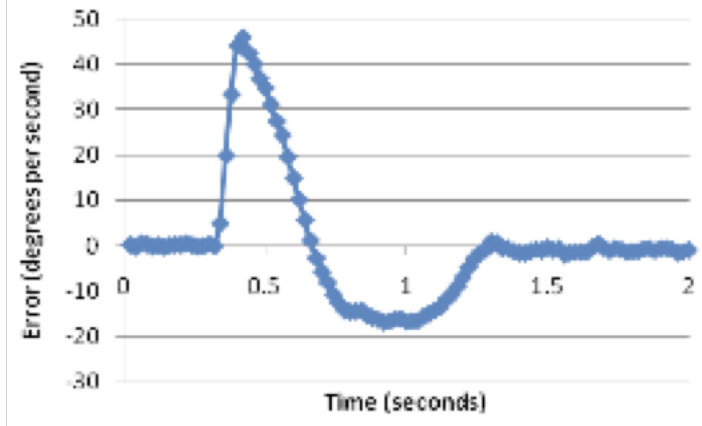

Figure 10: Impulse response of the one-axis gyroscope in the UUVs

An operational version of the autopilot unit was attached to one of the SCUUL UUVs and placed in the tank to determine its performance capabilities. The submarine was preprogrammed to travel in a downward spiral at a given angular rate. This test was also used to evaluate the quality of data produced by the Qualisys motion capture system. Figure 11 shows a visual representation of the 6DOF tracking data using a visualization developed in Matlab.

While the vehicle did perform a downward spiral, the center of the spiral tended to drift. This may have been due to the increased drag on the vehicle from the addition of the AU, which caused the vehicle to drift while turning.

Once the performance of the AU was determined, the ability of the motion capture system to identify and track mul-

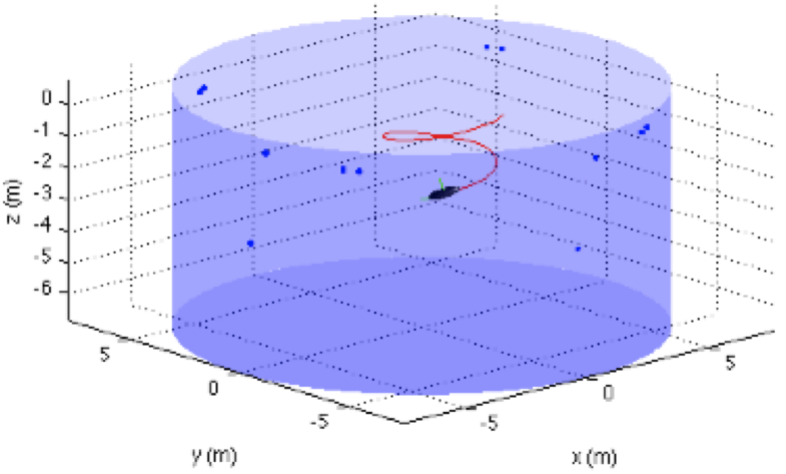

Figure 11: Motion capture tracking of one submarine under autopilot control is followed.

tiple bodies was assessed. Two subs under RC control were placed in the tank to determine the performance. Figure 12 shows the $6 \mathrm{DOF}$ tracking data for the collected data set.

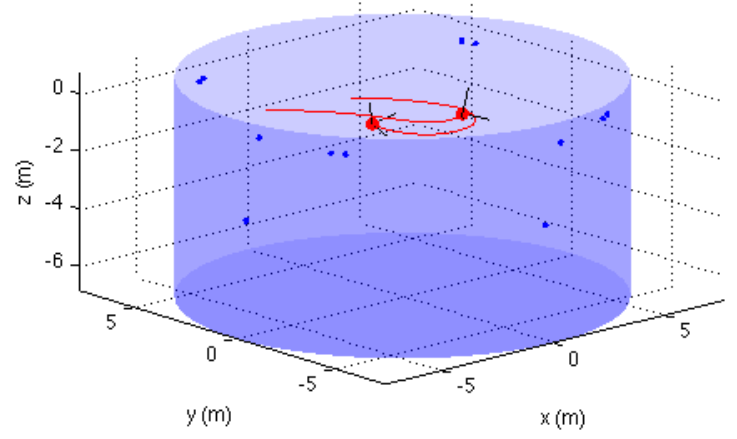

Figure 12: Motion capture tracking of two UUVs.

Even in the case where there are multiple objects in the water, the Qualisys system is able to track the submarines with sub-centimeter accuracy. The only condition placed on the UUV is that its marker configuration be unique from any other objects in the tank.

\subsection{Ongoing Work}

It was determined during the testing of the AU that attaching a pressure vessel to the bottom the UUVs greatly increases the drag on the vehicle, thereby reducing the speed. This introduces effects in the vehicle dynamics, such as roll/yaw coupling and turning drift. Since most of the control algorithms currently being tested in SCUUL use a self-propelled particle model for the underlying dynamics this may cause the heading-rate control law to produce undesired results.

Current work is focusing on moving the autopilot electronics to the main pressure vessel. The new architecture, shown in Figure 13, will place an ArduPilot controller, made by DIY Drones, and the gyroscope in the MPV of the submarine. Control commands from the Qualisys system will 
be sent directly to the UUVs onboard receiver, eliminating the need for a transmitter on the vehicle. These components can be powered from the battery already inside the UUV. With this new architecture, there is no need for a separate $\mathrm{AU}$, it is instead integrated into the MPV. The ArduPilot and the gyroscope are shown below.

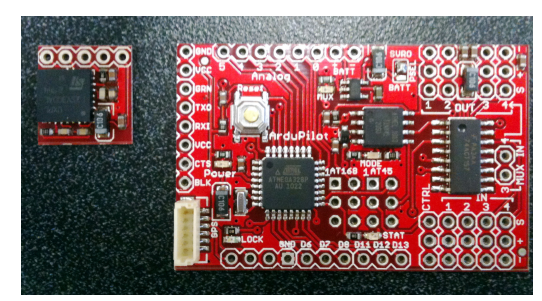

Figure 13: The new gyroscope (left) and ArduPilot (right).

In addition, it was determined that the fleet would be more versatile if the automatic depth controller was upgraded to a custom version designed by CDCL. The new architecture includes a pressure sensor which is attached directly to the ArduPilot. The ArduPilot can use the pressure reading in a proportional controller to adjust the depth of the vehicle. The desired depth can be hard-coded or it can be sent via the PCTx interface. This architecture is also shown in Figure 14.

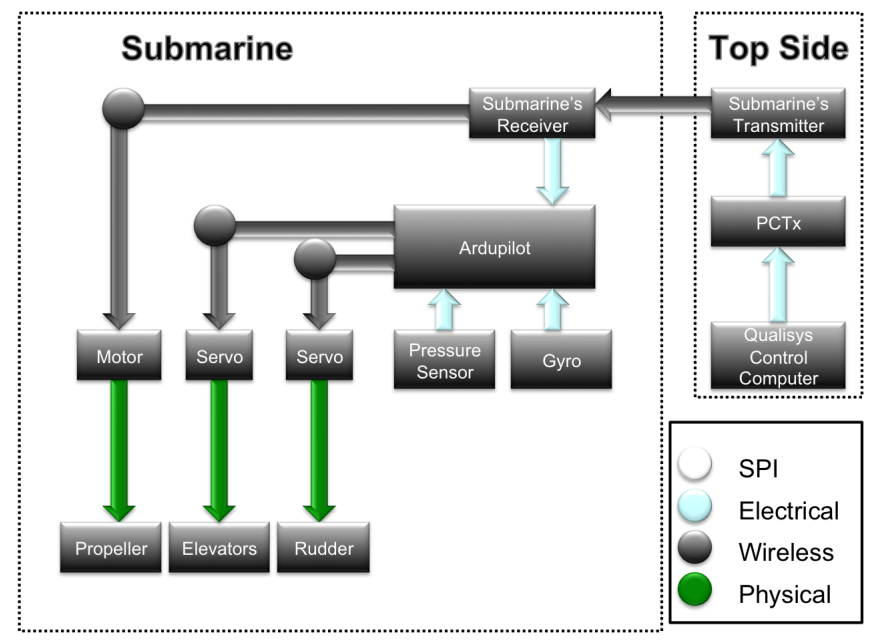

Figure 14: Future interface between the UUVs and top side operations.

This improved architecture increases the accuracy of the model used for the cooperative control laws that are currently being implemented in SCUUL and will therefore lead to enhances capabilities for test and evaluation of motion coordination algorithms for underwater vehicles.

\section{CONCLUSIONS}

This paper describes the University of Maryland SCUUL testbed. The testbed consists of six, propellor-driven UUVs, a 367,000 gallon dive tank, and a state-of-the-art underwater motion capture system. The current architecture has an autopilot unit attached to the bottom of each submarine through which closed-loop control of the vehicle can be performed. The motion capture system outputs data in real time. The data is processed to generate a desired heading rate to be sent to the submarines.

So far, the performance of the autopilot unit has been examined in and out of the water. An ArduPilot will be placed in the main pressure vessel of each submarine and will receive commands directly from the submarines receiver. Tests have also been conducted to examine the ability of the motion capture system to handle multiple rigid bodies. Preliminary results show that the system can handle at least two rigid bodies provided the placement reflective markers is unique to each UUV.

Efforts are currently underway to examine the ability to inject controlled flow fields into the NBRF. This would allow the simulation of real underwater flows in a controllable environment. With this architecture, the SCUUL testbed provides an environment where collective control laws for UUVs can be tested and evaluated in a safe and predictable environment.

\section{ACKNOWLEDGMENTS}

The authors would like to acknowledge the University of Maryland Space Systems Laboratory for supporting our underwater testing and maintaining the NBRF. We would also like to thank Levi DeVries, Colin Parker, Steve Sherman, Patrick Mohl, and Patrick Nolan for their continued support in submarine trials. We want to acknowledge the efforts of all past and present CDCL members for building and maintaining the UUV fleet. This work is supported by Army contract W9124Q09P0230 as part of the Unmanned Autonomous Systems Test program.

\section{REFERENCES}

[1] G. Hoffman, S. Waslander, and C. Tomlin. Mutual information methods with particle filters for mobile sensor network control. In Proceedings of the 45th IEEE Conference on Decision and Control, pages 1019-1024, 2006.

[2] E. W. Justh and P. S. Krishnaprasad. Equilibria and steering laws for planar formations. Syst Control Lett, 52(1):25-38, 2004.

[3] C. Peterson and D. A. Paley. Multi-vehicle coordination of autonomous vehicles in an unknown flowfield. In Proc. AIAA Conf. Guidance, Navigation, and Control (electronic), number AIAA-2010-7585,, August 2010.

[4] R. Sepulchre, D. Paley, and N. E. Leonard. Stabilization of planar collective motion: All-to-all communication. IEEE Trans. Automatic Control, 52(5):811-824, 2007. 